\title{
Modelación del volumen fustal de Pinus durangensis en Guachochi, Chihuahua, México
}

\author{
Modeling bole volume of Pinus durangensis in Guachochi, \\ Chihuahua, México
}

\author{
Marín Pompa-García1*, Ciro Hernández², \\ José Ángel Prieto-Ruiz 3 y Raymundo Dávalos Sotelo4
}

\begin{abstract}
RESUMEN
Pinus durangensis es la especie arbórea de mayor uso y distribución en la región de Guachochi, Chihuahua, México; sin embargo, el conocimiento para estimar con veracidad su volumen es limitado, por lo cual en este trabajo se planteó modelar el ahusamiento de dicha especie que posibilite generar una ecuación de volumen comercial compatible garantizando su aplicación operativa. Con ese propósito, se ajustó un modelo trigonométrico a 4502 pares de datos de 663 árboles derribados en operaciones de aprovechamiento forestal. La prueba de bondad de ajuste consistió en la comparación de estadísticos como el coeficiente de determinación, la raíz del error medio cuadrático y un índice de precisión. El ajuste incluyó el uso de métodos estadísticos apropiados para detectar y corregir los problemas de autocorrelación y heterocedasticidad asociados al desarrollo de ecuaciones de ahusamiento. El modelo ajustado arrojó buenos resultados en su capacidad predictiva y mediante su integración matemática se obtuvo una ecuación de ahusamiento compatible con los volúmenes comercial y total. Se concluyó que las ecuaciones generadas tienen un sustento estadístico sólido, por lo cual son una herramienta fundamental para mejorar la precisión en los inventarios forestales, asegurando así su aplicación no sólo en la región de estudio, sino que también se recomienda su puesta en práctica en otras localidades, mediante las adaptaciones a las que haya lugar.
\end{abstract}

PALABRAS CLAVE:

Ahusamiento, Chihuahua, modelo trigonométrico, volumen.

\begin{abstract}
Pinus durangensis is the most utilized and abundant tree species in the Guachochi region of the state of Chihuahua; however, there is a lack of appropriate knowledge to get accurate volume estimations. Therefore, the purpose of this study was to model the tapering of the bole to allow developing an equation to predict merchantable and total volume, which guarantees its operative application. For this purpose, a trigonometric model was fitted to 4502 data pairs of 663 trees felled during harvesting operations. The goodness of fit test consisted of comparing the statistics, coefficient of determination, root mean square error, and a precision index. Model fitting includes the use of appropriate statistical procedures to account for the problems of autocorrelation and heterocedasticity associated with the
\end{abstract}

Facultad de Ciencias Forestales. Universidad Juárez del Estado de Durango. Río Papaloapan y Blvd. Durango s/n Col. Valle del Sur. Tel (618) 130-10-96. Ce: mpgarcia@ujed.mx Instituto de Silvicultura e Industria de la Madera. Universidad Juárez del Estado de Durango. Blvd. Guadiana 501; CP 34120, Durango, Dgo., México.

3 Instituto Nacional de Investigaciones Forestales Agrícolas y Pecuarias; CEVAG-Durango; Km. 5.5 carretera al Mezquital; Durango, Dgo., México.

4 Instituto de Ecología, A.C., km. 2.5 carretera antigua a Coatepec No. 351, Congregación "El Haya”, Xalapa, Ver. C.P. 91070 , México.

* $\quad$ Autor para correspondencia. 
development of taper equations. The model gave best results in terms of predictive capacity, and via mathematic integration, an equation for tapering was obtained which is compatible with the merchantable and the total volume. It was concluded that the generated equations have a solid statistical foundation, and therefore constitute an essential tool for improving accuracy in forestry inventories, assuring its usage not only in the study region but its application is recommended also in other sites, with the necessary adaptation of the model to the respective local conditions.

PALABRAS CLAVE:

Chihuahua, Taper, trigonometric model, volume.

\section{INTRODUCCIÓN}

En el estado de Chihuahua existen grandes superficies de bosques de pino cuyo aprovechamiento constituye la principal fuente de ingresos para los silvicultores. Particularmente, en el área de influencia de la organización Silvicultores Unidos de Guachochi, S.C., en el sur de Chihuahua, la importancia de Pinus durangensis es sobresaliente, pues se distribuye en $80 \%$ de la superficie arbolada y genera un volumen aprovechable anual del orden de los $190000 \mathrm{~m}^{3}$ (SEMARNAT, 2005), el cual se industrializa para obtener productos de alto valor comercial, como madera contrachapada y aserrío, principalmente. Sin embargo, es limitado el conocimiento sobre la veracidad de las estimaciones del volumen de esta especie, dado que en los programas de manejo de la región se utilizan ecuaciones desarrolladas a nivel de género, obtenidas incluso para otras localidades y sin mayor sustento estadístico (SUG, 2007); específicamente, según información de la dirección técnica, se ha venido utilizando el modelo de Biging (1984), que se ha empleado para la misma especie en regiones similares pero presenta desventajas en la estimación de diámetros para categorías mayores de $30 \mathrm{~cm}$, sugiriéndose investigación adicional (Corral et al., 1999). Por lo anterior, es imperativo contar con herramientas confiables para estimar volúmenes de madera comerciales y totales, a partir de ecuaciones que modelen el ahusamiento fustal; estas herramientas deben estar fundamentadas en procedimientos estadísticos rigurosos, para garantizar su aplicación operativa de acuerdo a las exigencias que marca la legislación forestal vigente.

Dentro de la dasonomía existe un alto grado de desarrollo en la modelación. El empleo de ecuaciones de ahusamiento es un buen ejemplo de ello. A través del uso de metodologías adecuadas, particularmente de los procedimientos de regresión y de la modelación matemática, se han logrado considerables reducciones de costos en los inventarios, sin tener que disminuir la precisión de los estimadores en la búsqueda de optimizar la distribución de productos forestales maderables previo a su utilización (Pompa y Solís, 2008).

Existen numerosos esfuerzos para modelar el ahusamiento de los fustes como apoyo para estimar su volumen comercial; en los últimos treinta años se han estudiado desde los modelos más simples de ahusamiento (Kozak et al., 1969; Ormerod, 1973; Hilt, 1980), modelos polinomiales segmentados (Bruce et al., 1968; Max and Burkhart, 1976; Cao et al., 1980) y modelos geométricos y trigonométricos (Parresol y Tomas, 1996; Fang y Bailey, 1999; Zhang et al., 2002; Bi, 2000). Sin embargo, Newnham (1998) menciona que hay al menos dos razones para continuar con el estudio de este tema: 1) hasta hoy no existe una teoría que explique adecuadamente la variación de la forma del fuste para todos los árboles y 2) las ecuaciones de ahusamiento pueden ser una herramienta eficiente para estimar tanto el 
volumen comercial como el volumen total del fuste. Así, Clutter (1980) notó que en una ecuación dada de volumen comercial está intrínsecamente definida una función de ahusamiento, lo cual a su vez, mediante integración matemática, posibilita obtener una ecuación compatible de volumen total.

Recientemente se ha demostrado la gran utilidad de usar modelos trigonométricos (Bi, 2000; Bi y Long, 2001), ya que estos pueden ajustarse con gran precisión a datos procedentes de diferentes especies y formas, dado que el punto de inflexión varía de acuerdo al tamaño del árbol, situación que se diferencia de los modelos típicos de forma o exponente variable.

En general, los modelos de ahusamiento deben cumplir con las siguientes características: 1) cuando la altura parcial es igual a la altura total, el diámetro predicho debe ser cero; 2) a $1.3 \mathrm{~m}$ de altura debe predecir el diámetro normal y 3) debajo de $1.3 \mathrm{~m}$ de altura debe predecir diámetros mayores al diámetro normal.

\section{OBJETIVO}

El objetivo en este trabajo fue modelar el ahusamiento para Pinus durangensis, que permita, en consecuencia, generar ecuaciones de volumen comercial y total en los bosques de la organización Silvicultores Unidos de Guachochi, Chihuahua, México.

\section{MATERIALES Y MÉTODOS}

\section{Descripción del área de estudio}

El área de estudio corresponde a la región administrativa de Silvicultores Unidos de Guachochi, S.C., cuya superficie es de 767000 ha y se localiza en el suroeste del estado de Chihuahua, entre los paralelos 27052' y $27^{\circ} 30^{\prime}$ latitud norte y los meridianos 106030' y 107030 longitud oeste (Figura 1). Comprende una parte de los municipios de Urique, Batopilas, Balleza y Guachochi.

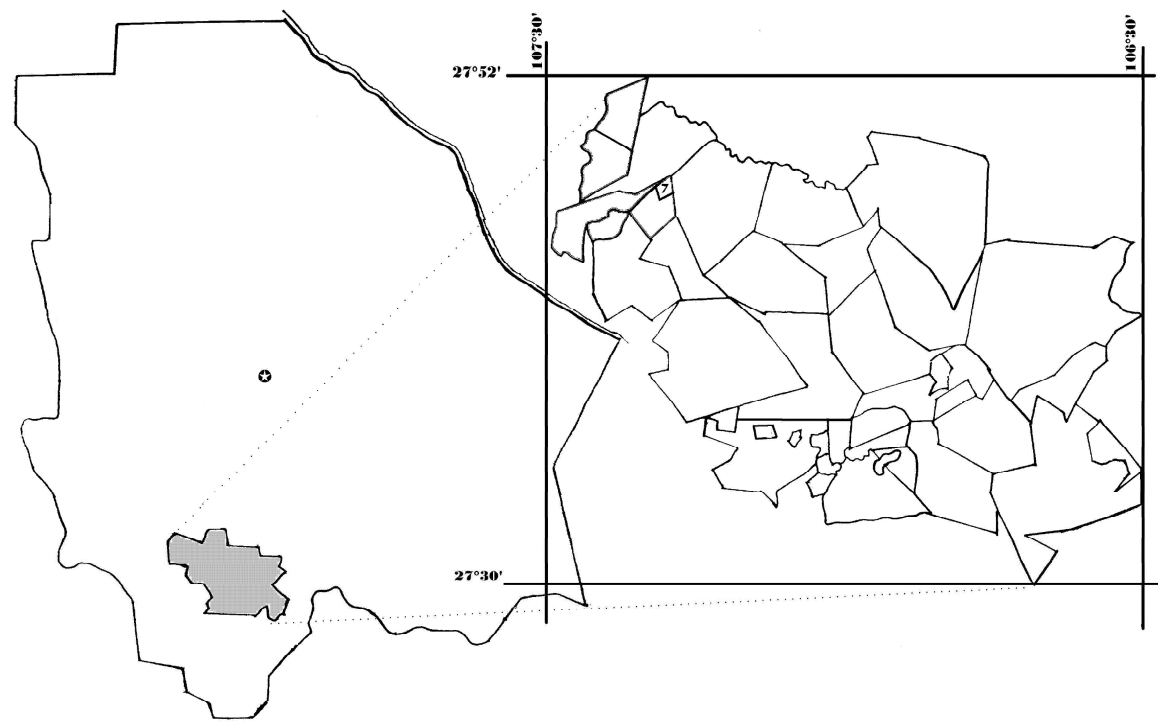

Figura 1. Localización del área de estudio. 
La Organización está integrada por 30 ejidos y 1000 pequeñas propiedades, con una población de 110000 beneficiarios, de los cuales $85 \%$ corresponde a la población Rarámuri (INEGI, 2004). Esta organización forestal se encuentra enclavada en lo alto de la Sierra Madre Occidental, conocida como Sierra Tarahumara, con terrenos bastante accidentados que presentan elevaciones y depresiones muy irregulares (Pompa, 1995).

La vocación del uso del suelo es forestal; existe un potencial productivo de madera de $250000 \mathrm{~m}^{3}$ de pino y $80000 \mathrm{~m}^{3}$ de encino por año (SEMARNAT, 2005). El bosque de esta región es de clima templado y semifrío con diferentes grados de humedad; predominan los géneros: Pinus, Quercus, Fraxinus y Juniperus, entre otros (SEMARNAT, 2005).

\section{Descripción de la población de estudio}

La población de estudio correspondió a las masas forestales arboladas donde vegeta Pinus durangensis dentro del área de influencia de Silvicultores Unidos de Guachochi, empresa de productores concesionaria de los servicios técnicos forestales, delimitándose mediante el uso de la cartografía disponible en la dirección técnica sobre tipos de vegetación contenida en los programas de manejo de la zona.

\section{Descripción de la muestra}

Se tomó una muestra aleatoria de 663 árboles para representar todas las calidades de estación del área de estudio, así como también la distribución de los árboles en términos de clases de diámetro y altura (Figura 2a). La toma de datos de campo se realizó mediante un muestreo destructivo aprovechando las áreas de corta del año 2003, para lo cual se midieron las siguientes variables: diámetro normal con corteza $(D$, en $\mathrm{cm})$, altura total $(H$, en $\mathrm{m})$, diámetro con corteza para cada sección $(d$, en $\mathrm{cm})$ y la altura de cada sección con respecto al suelo ( $h$ en $\mathrm{cm}$ ). Se integraron 4502 pares de datos $(d$ y $h$ ) distribuidos a lo largo del tronco. En la tabla 1 se muestra un resumen de las estadísticas descriptivas de los árboles utilizados.

\section{Donde:}

$$
D=\text { diámetro con corteza a la altura }
$$
del pecho $(1,3 \mathrm{~m}$ en $\mathrm{cm})$.

$H=$ Altura total $(\mathrm{m}) ; h=$ altura de la sección del árbol a partir del suelo (en $\mathrm{m}$ ). $d=$ diámetro con corteza $(\mathrm{en} \mathrm{cm}) \mathrm{a}$ la altura $h$ (en $\mathrm{m})$.

Tabla 1. Estadísticas descriptivas de los árboles utilizados.

\begin{tabular}{cccccc}
\hline Variable & $\begin{array}{c}\text { Número de } \\
\text { observaciones }\end{array}$ & Media & $\begin{array}{c}\text { Desviación } \\
\text { estándar }\end{array}$ & Máximo & Mínimo \\
\hline$D$ & 663 & 38 & 10,7 & 66 & 10,2 \\
$d$ & 4502 & 25,9 & 16,2 & 84 & 0 \\
$H$ & 663 & 18,9 & 5,8 & 36,2 & 4,2 \\
$h$ & 4502 & 9,1 & 7,75 & 36,2 & 0,1 \\
\hline
\end{tabular}




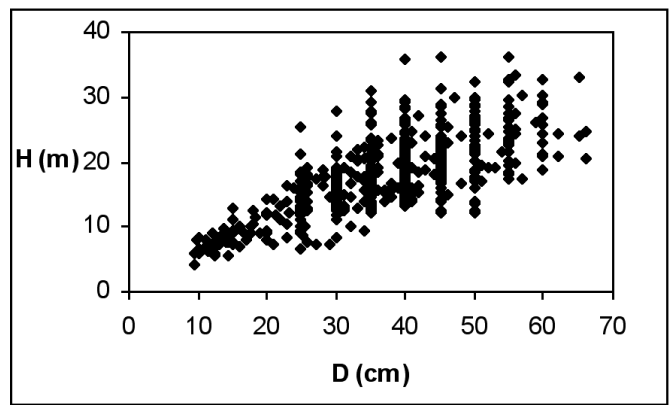

a)

b)

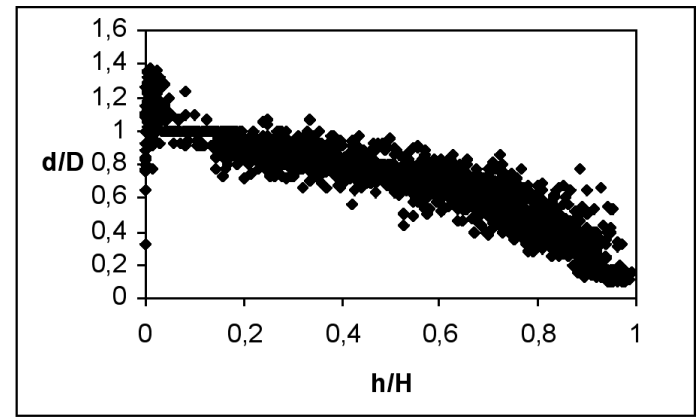

Figura 2. Dispersión de observaciones diámetro normal - altura (a). Dispersión de alturas relativas respecto a diámetros relativos de los 663 árboles utilizados en el ajuste del modelo trigonométrico (b).

La figura $2 b$ representa las alturas relativas $(h / H)$ frente a los diámetros relativos $(d / D)$ de cada sección medida del tronco. La amplitud de los datos refleja la magnitud de la forma de los árboles que conformaron la muestra utilizada.

\section{Modelo probado}

Para tratar de predecir el perfil de los árboles, con base en los datos provenientes de la muestra del área de estudio, se probó el modelo trigonométrico de $\mathrm{Bi}$ (2000). Su ecuación simplificada es $d r=B K$, donde $d r$ es el diámetro relativo del fuste a una altura relativa dada, $B$ es la base del modelo en función de la altura relativa y de las características dendrométricas del árbol (diámetro normal y altura total), y $K$ es una función que describe los cambios de la forma del fuste y sus características dendrométricas. La ecuación de base $(B)$ ha sido construida a partir de ecuaciones trigonométricas de proporción del volumen según la geometría del fuste. Gracias a su flexibilidad, este modelo se ha utilizado para describir el perfil del fuste de Eucalyptus $s p$. en Australia (Bi, 2000), Pinus radiata en New South Wales (Bi y Long, 2001) y recientemente en Populus $x$ euramericana en Navarra (Rodríguez y Molina, 2003), pero se desconocía su bondad de ajuste para Pinus durangensis. Su expresión matemática corresponde a: 


$$
d=D\left[\frac{\ln \operatorname{sen}\left(\frac{\pi}{2} \cdot \frac{h}{H}\right)}{\ln \operatorname{sen}\left(\frac{\pi}{2} \cdot \frac{1.3}{H}\right)}\right] \beta^{\beta_{0}+\beta_{1} \cdot \operatorname{sen}\left(\frac{\pi}{2} \cdot \frac{h}{H}\right)+\beta_{2} \cdot \cos \left(\frac{3 \cdot \pi}{2} \cdot \frac{h}{H}\right)+\beta_{3} \cdot \frac{\operatorname{Sen}\left(\frac{\pi}{2} \cdot \frac{h}{H}\right)}{\frac{h}{H}}+\beta_{4} \cdot D+\beta_{5} \cdot \frac{h}{H} \cdot \sqrt{D}+\beta_{6} \cdot \frac{h}{H} \cdot \sqrt{H}}
$$

\section{Donde:}

$$
d \text { = diámetro con corteza que se }
$$
alcanza a la altura $h$, en $\mathrm{cm}$.

$h=$ altura desde la base del árbol hasta donde se alcanza el diámetro $d$, en $\mathrm{m}$. en $\mathrm{cm}$.

$D=$ diámetro normal con corteza,

$H=$ Altura total del árbol, en $\mathrm{m}$.

$i=$ Coeficientes de regresión a determinar mediante el ajuste.

Los cuatro primeros parámetros de la función $K\left(\begin{array}{lll}\beta_{0} & a & \beta_{3}\end{array}\right)$ describen los cambios de la forma del fuste a lo largo de éste, mientras que los tres últimos $\left(\beta_{4}\right.$ a $\beta_{6}$ ) caracterizan la forma del fuste dependiendo de las características dendrométricas del árbol evaluado.

Este modelo cumple con la condición de que $d=0$ cuando $H=h$ y pueden ser usados para predecir diámetros $(d)$ a cualquier altura del trozo $(h)$, y volumen entre dos diámetros o alturas del tronco.

\section{Ajuste del modelo}

El ajuste del modelo se realizó por el método de mínimos cuadrados ordinarios, empleando el programa estadístico SAS/STAT, (SAS Institute Inc., 2004). AI desarrollar procedimientos de regresión, usualmente se asume que el error es independiente y que se distribuye normalmente con varianza y media igual a cero
(Gujarati, 2004). Sin embargo, la heterocedasticidad y la autocorrelación son los inconvenientes más importantes en el caso de modelos de ahusamiento (Kozak, 1997). Por tanto, en este trabajo se utilizaron métodos generalizados de regresión lineal y no lineal para modelar la estructura del error. Los trabajos de Monserud (1984), Goelz y Burk (1992, 1996, 1998) y Huang (1997) presentan aplicaciones prácticas de estos métodos. Los valores de la prueba de Park "P" (Park, 1966), y el estadístico de Durbin y Watson "DW" (Durbin y Watson, 1951), evidenciaron que la estimación final de los parámetros no estuvo afectada por problemas de heterocedasticidad y autocorrelación, respectivamente.

Los criterios empleados para evaluar la bondad de ajuste del modelo se basaron en un análisis numérico y gráfico de los residuos, es decir, la diferencia entre el diámetro medido y el predicho. El análisis numérico consistió en la comparación de tres estadísticos utilizados con frecuencia en el ajuste de modelos: 1) el sesgo ( $\bar{E}$ ), que evalúa la desviación del modelo con respecto a los valores observados; 2) la raíz del error medio cuadrático $(R E M C)$, que analiza la precisión de las estimaciones, y 3) el coeficiente de determinación ajustado $\mathrm{R}^{2}$ adj que representa la parte de la varianza explicada por el modelo, teniendo en cuenta el número de parámetros de éste. Sus expresiones son: 
Sesgo:

Raíz del error medio cuadrático:

Coeficiente de determinación ajustado:

Donde $\mathrm{y}_{i}, \hat{y}_{i}, \bar{y}$ son el valor observado, predicho y medio de la variable dependiente, respectivamente; $n$ es el número total de observaciones usadas para ajustar el modelo; y $p$ es el número de parámetros del modelo.

Para el análisis gráfico se examinaron diferentes representaciones de los datos experimentales y de los residuos después del ajuste de los modelos. Estos gráficos representan una herramienta muy importante en la selección de un modelo, ya que permiten detectar errores o comportamientos anormales (Rawlings, 1988; Neter et al., 1990).

\section{RESULTADOS}

En la tabla 2 se presentan los valores de los parámetros estimados y de los estadísticos del ajuste del modelo. Todas las estimaciones de los parámetros resultaron ser significativas al $95 \%$. De acuerdo con los resultados mostrados en la tabla 2, el modelo explica de manera adecuada el perfil del árbol, ya que presenta valores bajos del REMC, explicando el $95,5 \%$ de la varianza del diámetro $(d)$.

Con relación a la heterocedasticidad, el gráfico de los residuos frente a los valores predichos por el modelo (Figura 3) y la prueba de Park (1966) evidenciaron que existe igualdad de varianza y que no hay relación estadísticamente significativa de los residuales
$\bar{E}=\sum_{i=1}^{n}\left(y_{i}-\hat{y}_{i}\right) / n$

$R E M C=\sqrt{\sum_{i=1}^{n}\left(y_{i}-\hat{y}_{i}\right)^{2} /(n-p)}$

$R_{\mathrm{adj}}^{2}=1-(n-1) \cdot \sum_{i=1}^{n}\left(y_{i}-\hat{y}_{i}\right)^{2} /(n-p) \cdot \sum_{i=1}^{n}\left(y_{i}-\bar{y}_{i}\right)^{2}$

con las variables explicatorias. El estadístico de Durbin y Watson es próximo a 2 $(D W=1.3926)$ y está dentro de los intervalos aceptados en las tablas respectivas para rechazar la existencia de autocorrelación entre los residuos con un $\alpha$ de 0,05 (Durbin y Watson, 1951).

Aunque estos estadísticos y sus supuestos son buenos indicadores de la bondad global de ajuste de la función de ahusamiento, es conveniente una representación gráfica por secciones relativas de altura en el árbol. Según Castedo (2003), el modelado por posición es muy importante para decidir si en realidad la función de ahusamiento debe ser recomendada. Con esa finalidad, en la figura 4 se ilustra el efecto que tiene el sesgo y el cuadrado medio del error a lo largo del fuste, para lo cual se grafica la conducta del modelo. En los gráficos referidos, es evidente la bondad de ajuste del modelo, ya que tiene una mejor estimación del perfil en la parte basal donde se sitúa la mayor parte del volumen comercial; bajo este criterio de operatividad práctica se considera que este modelo es definitivamente un buen predictor de diámetros a diferentes alturas del fuste y por ende, un buen descriptor del ahusamiento de Pinus durangensis de la región de estudio. Su buen comportamiento estriba en su gran flexibilidad, ya que la altura normal relativa $(1.3 / \mathrm{h})$ varía con la altura total del árbol y las características del exponente están relacionadas con la forma a lo largo del tronco y consideran las diferencias 
Tabla 2. Comparación del ajuste de modelos de ahusamiento (con un nivel de significancia $\operatorname{Pr}>|t|<0.0001)$.

\begin{tabular}{|c|c|c|c|c|c|c|}
\hline & \multirow[t]{2}{*}{ Parámetro } & \multirow[t]{2}{*}{ Estimación } & \multirow{2}{*}{$\begin{array}{l}\text { Error } \\
\text { estándar }\end{array}$} & \multicolumn{3}{|c|}{ Estadisticos del ajuste } \\
\hline & & & & $\bar{E}$ & $R E M C$ & $R_{a d j}^{2}$ \\
\hline \multirow{7}{*}{ Bi (2000) } & $\beta_{0}$ & 1,292657 & 0,0640 & \multirow{7}{*}{0,2052} & \multirow{7}{*}{8,117} & \multirow{7}{*}{0,955} \\
\hline & $\beta_{1}$ & $-0,39074$ & 0,0351 & & & \\
\hline & $\beta_{2}$ & $-0,02798$ & 0,0714 & & & \\
\hline & $\beta_{3}$ & $-0,65114$ & 0,0402 & & & \\
\hline & $\beta_{4}$ & 0,000833 & 0,00028 & & & \\
\hline & $\beta_{5}$ & 0,008942 & 0,00571 & & & \\
\hline & $\beta_{6}$ & $-0,01471$ & 0,00293 & & & \\
\hline
\end{tabular}

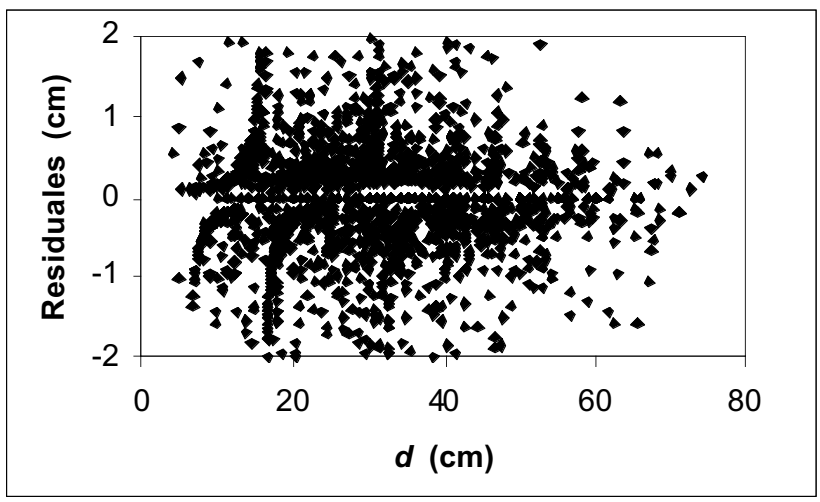

Figura 3. Gráfico de residuos frente a valores predichos del modelo de Bi (2000). 

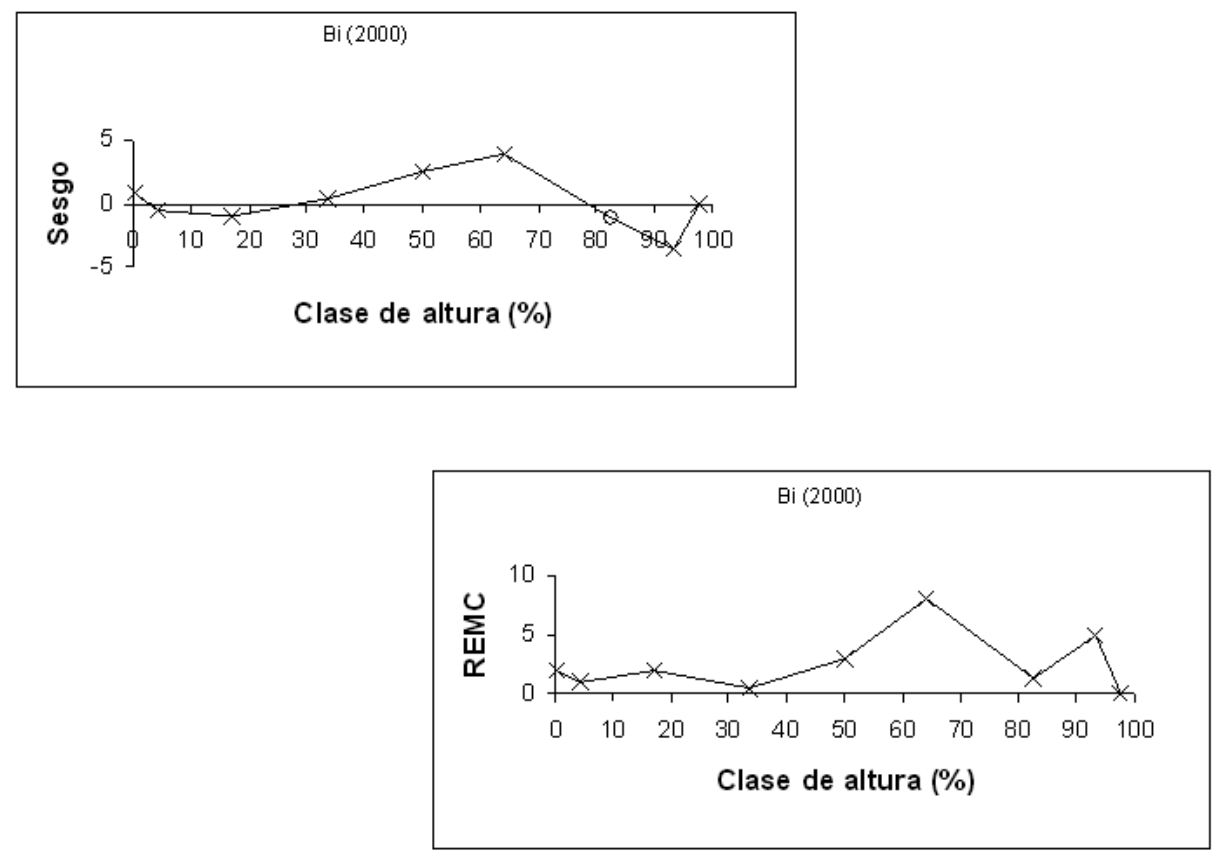

Figura 4. Comportamiento del sesgo y el REMC a lo largo del fuste para la función de ahusamiento de $\mathrm{Bi}(2000)$.

entre árboles de distintos tamaños, lo cual es correspondiente a los resultados encontrados por varios autores para especies de pino (Castedo, 2003; Rodríguez y Molina, 2003).

En la figura 5 se presenta la bondad de ajuste del modelo para los valores observados de $d$ a lo largo del fuste de un árbol seleccionado al azar. Se puede apreciar que el modelo simula satisfactoriamente el perfil en la mayor parte del fuste, disminuyendo ligeramente su consistencia en la punta, donde se presenta un ahusamiento difícil de modelar debido a factores como el desarrollo de la copa, calidad del sitio y la densidad (Larson, 1963).

Con base en los resultados discutidos, en la ecuación definida para la especie en estudio queda implícito el índice de esbeltez del fuste $(H / D)$, mediante su inverso, es decir, $D / H$, como una medida de la proporcionalidad de esas dimensiones. Además, para cuando $h=H$, el diámetro estimado es cero, como lo indica la suma de sus coeficientes, cumpliendo satisfactoriamente con esta propiedad.

\section{DISCUSIÓN}

El modelo de $\mathrm{Bi}$ (2000) recomendado para Pinus durangensis en este estudio, tiene una importante ventaja comparativa con respecto al trabajo de Corral et al. (1999). En primer lugar, es consistente en sus estimaciones a lo largo del perfil del árbol, mientras que en la publicación referida se manifiesta la 


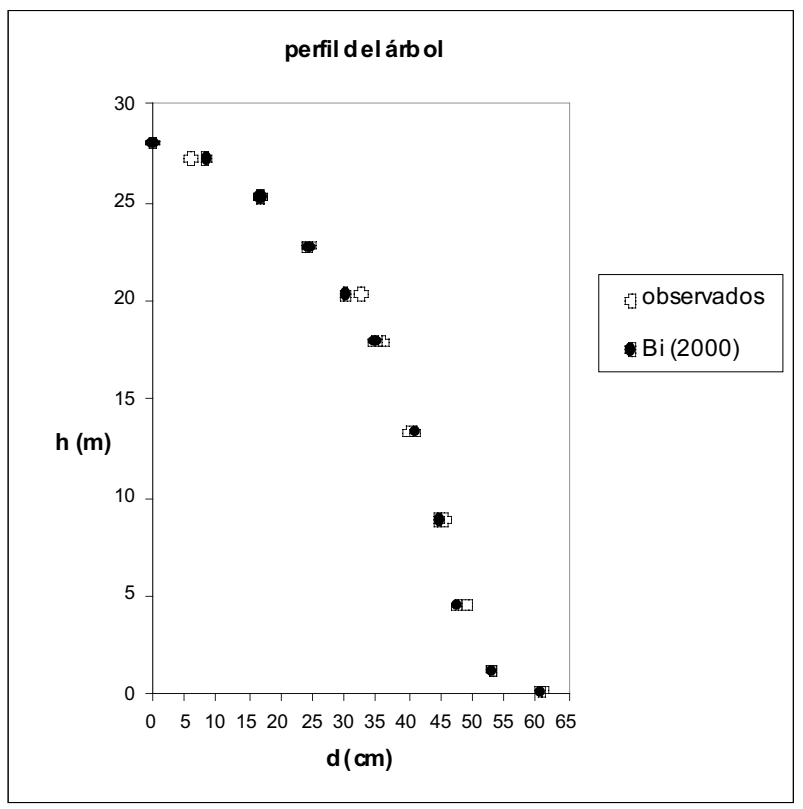

Figura 5. Gráfico del ajuste al perfil de un árbol obtenido con el modelo de "Bi (2000)" propuesto para Pinus durangensis.

presencia exponencial de errores para diámetros mayores a $30 \mathrm{~cm}$ con sus efectos multiplicativos en el cálculo erróneo del volumen, por lo cual los mismos autores recomiendan estudiar la constante de su modelo para mejorar el ajuste, el cual solamente contiene dos parámetros en su expresión, en contraste con el modelo aquí sugerido, cuya expresión es más compleja, aunque su integración numérica no representa mayor dificultad para las modernas técnicas computacionales disponibles, como lo ha demostrado Castelo (2003) mediante el procedimiento QUAD del SAS/STAT ${ }^{\circledR}$ (SAS Institute Inc., 2004).

Otra aportación importante del modelo es su compatibilidad con una ecuación de volumen (p. e., Smalian); es decir, cuando esta función es integrada genera estimaciones de volumen comer- cial y total idénticas a las obtenidas por una ecuación de volumen existente (Demaerschalk, 1972; Avery y Burkhart, 1983). Sin embargo, debe considerarse que los volúmenes reales generalmente no se conocen, sino que suelen calcularse por las fórmulas de Smalian, Huber o Newton.

\section{CONCLUSIONES}

El modelo de $\mathrm{Bi}$ (2000) presentó buena capacidad predictiva para describir el perfil del fuste de Pinus durangensis en la parte suroeste del estado de Chihuahua. Este modelo puede ser usado confiablemente para predecir el diámetro a cualquier altura del fuste. Dicho modelo tiene la importante ventaja de ser una función de ahusamiento consistente en sus estimaciones a lo largo del perfil fustal; además, es compatible con las 
ecuaciones de volumen existentes para el área de estudio. La ecuación generada en este trabajo constituye una herramienta valiosa para ser aplicada en los inventarios forestales en la región de estudio y tiene un sustento estadístico sólido, por lo que también se recomienda su aplicación en otras regiones similares, con las adaptaciones locales a las que haya lugar.

\section{RECONOCIMIENTOS}

Se reconoce el apoyo otorgado por Silvicultores Unidos de Guachochi, S.C., quienes motivados por generar una herramienta actualizada para sus inventarios forestales, apoyaron en la toma de datos utilizados en el presente estudio.

\section{REFERENCIAS}

Avery, T.E. y H.E. Burkhart. 1983. Forest measurements. McGraw-Hill Co. USA. 330 p.

Bi, H. 2000. Trigonometric variable-form taper equations for Australian eucalyptus. Forest Science 46(3): 397-409.

Bi, H. y Long Y. 2001. Flexible taper equation for site-specific management of Pinus radiate in New South Wales, Australia. Forest Ecology Management 148: 79-91.

Biging, G.S. 1984. A compatible volumetaper function for Alberta trees. Forest Science 14: 339-350.

Bruce, D., R.O. Curtis y C. Vancoevering. 1968. Development of system and taper volume tables for red alder. Forest Science 14: 339-350.

Cao, Q.V., H.E. Burkhart E. y T.A. Max. 1980. Evaluation of two methods for cubic-volume prediction of loblolly pine to any merchantable limit. Forest Science 26(1): 71-80.

Castedo, D.F. 2003. Modelo dinámico de crecimiento para las masas de Pinus radiata D. Don en Galicia. Tesis doctoral. Universidad de Santiago de Compostela, España. 297 p.

Clutter, J.L. 1980. Development of taper functions from variable-top merchantable volumen equations. Forest Science 26: 117-120.

Corral, R.S., Návar J.J., Fernandes S.F. 1999. Ajuste de funciones de ahusamiento a los perfiles fustales de cinco pináceas de la región de $\mathrm{E}$ Salto, Durango. Madera y Bosques 5: 53-65.

Demaerschalk, J.P. 1972. Converting volume equations to compatible taper equations. Forest Science 18(3): 241-245.

Durbin, J. y Watson G.S. 1951. Testing for serial correlation in least squares regression. Biometrika, Vol. 38: 159-177.

Fang, Z. y Bailey. 1999. Compatible volume and taper models with coefficients for tropical species on Hainan Island in southern China. Forest Science 45: 85-100.

Goelz, J.C.G. y Burk T.E. 1992. Development of a well-behaved site index equation: jack pine in north central Ontario. Canadian Journal of Forest Research 22: 776-784.

Goelz, J.C.G. y Burk T.E. 1996. Measurement error causes bias in site index equations. Canadian Journal of Forest Research 26:1585-1593. 
Goelz, J.C.G. y Burk T.E. 1998. Long-term trends in height growth of jack pine in north central Ontario. Forest Science 44: 158-164.

Gujarati, D. 2004. Econometría. McGrawHill Interamericana de México, México, D.F. 972 p.

Huang, S. 1997. Development of a subregion-based compatible height-site index-age model for black spruce in Alberta. Alberta Land and Forest Service, For. Mgmt. Res. Note No 5, Pub No. T/352, Edmonton, Alberta.

Hilt, D.E. 1980. Development of taper functions from variable-top merchantable volume equations. Forest Science 26: 117-120.

INEGI. 2004. Anuario Estadístico. Chihuahua. Carpetas de datos básicos estatales. Datos básicos de Chihuahua. Dirección General de Estadística. Disponible en: http//www.cuentame.inegi.gob.mx.

Kozak, A.D.D. Munro y J.H.G. Smith. 1969. Taper functions and their application in forest inventory. For. Chron. 45(4): 278-283.

Kozak, A. 1997. Effects of multicollinearity and autocorrelation on the variableexponent taper functions. Can. J. For. Res. 27: 619-629.

Larson, P.R. 1963. Stem form development of forest trees. Forest Science Monographs 5: 1-41.

Max, T.A. y H. E. Burkhart. 1976. Segmented polynomial regression applied to taper equations. Forest Science 22(3):283-289.

Monserud, R.A. 1984. Height growth and site index curves for inland Douglasfir based on stem analysis and forest habitat type. Forest Science 30: 943965.

Neter, J., Wasserman W. y Kutner M. 1990. Applied Linear Statistical Models. 3rd ed. Irwin, Homewood, IL.

Newnham, R.M. 1988. A variable-form taper function. Petawawa Nt. For. Inst. Information Rep. PI-X-83. 33 p.

Ormerod, D.W. 1973. A simple bole model. For. Chron. 49: 136-138.

Parresol, B.R. y C.C. Tomas. 1996. A simultaneous density-integral system for estimating stem profile and biomass: Slash pine and Willow Oak. Canadian Journal of Forest Research 26: 773-781.

Park, R.E. 1966. Estimation with heterocedastic error terms. Econometrica 34(4): 888

Pompa-G., M. 1995. Sistema de cubicación para el género Pinus en la UCODEFO \# 7. "NorogachiGuachochi”, Chihuahua. Tesis de Maestría. Colegio de Postgraduados, Montecillos, Méx. 105 p.

Pompa-G., M. y M.R. Solís. 2008. Ecuación de volumen para el género Quercus en la región noroeste de Chihuahua, México. Quebracho. Revista de Ciencias Forestales. En prensa.

Rawlings, J.O. 1988. Applied Regression Analysis -A Research Tool. Wadsworth, Belmont, CA.

Rodríguez, F. y Molina. F. 2003. Análisis de modelos de perfil del fuste $y$ estudio de la cilindricidad para tres clones de chopo (Populus $x$ euramericana) en Navarra. Invest. Agrar. Sist. Recur. For. 12(3): 73-85. 
SAS Institute Inc. 2004. SAS/ETS ${ }^{\circledR} 9.1$ User's Guide. SAS Institute Inc., Cary, NC.

SEMARNAT. 2005. Control técnico de aprovechamientos. Listado electrónico. Delegación federal de Chihuahua. Subdelegación de gestión para la protección ambiental y recursos naturales.
Silvicultores Unidos de Guachochi. 2007. Programa de manejo forestal del Ejido Guachochi. Memoria Técnica, $190 \mathrm{p}$.

Zhang, L., Peng L., Huang S. y Zhou, X. 2002. Development and evaluation of ecoregion-based tree heightdiameter models for jack pine in Ontario. For Chron. 78: 530-538. durangensis en Guachochi, Chihuahua, México. Madera y Bosques 15(1):61-73. 
Página en blanco intencionalmente (This page left blank intentionally) 